\title{
Alternating group covers of the affine line
}

\author{
Jeremy Muskat and Rachel Pries
}

June 5, 2022

\begin{abstract}
For an odd prime $p \equiv 2 \bmod 3$, we prove Abhyankar's Inertia Conjecture for the alternating group $A_{p+2}$, by showing that every possible inertia group occurs for a (wildly ramified) $A_{p+2}$-Galois cover of the projective $k$-line branched only at infinity where $k$ is an algebraically closed field of characteristic $p>0$. More generally, when $2 \leq s<p$ and $\operatorname{gcd}(p-1, s+1)=1$, we prove that all but finitely many rational numbers which satisfy the obvious necessary conditions occur as the upper jump in the filtration of higher ramification groups of an $A_{p+s^{-}}$ Galois cover of the projective line branched only at infinity.

2010 MSC: 11G20 and 12F12.
\end{abstract}

\section{Introduction}

Suppose $\phi: Y \rightarrow \mathbb{P}_{k}^{1}$ is a $G$-Galois cover of the projective $k$-line branched only at $\infty$ where $G$ is a finite group and $k$ is an algebraically closed field of characteristic $p>0$. Let $p(G) \subset G$ be the normal subgroup generated by the conjugates of a Sylow $p$-subgroup. Then the $G / p(G)$-Galois quotient cover is a prime-to- $p$ Galois cover of $\mathbb{P}_{k}^{1}$ branched only at $\infty$. Since the prime-to- $p$ fundamental group of the affine line $\mathbb{A}_{k}^{1}$ is trivial, this implies that $p(G)=G$; a group $G$ satisfying this condition is called quasi-p. In 1957, Abhyankar conjectured that a finite group $G$ occurs as the Galois group of a cover $\phi: Y \rightarrow \mathbb{P}_{k}^{1}$ branched only at $\infty$ if and only if $G$ is a quasi- $p$ group [1]. Abhyankar's conjecture was proved by Raynaud [10] and Harbater [7].

Now suppose $G_{0}$ is the inertia group at a ramified point of $\phi$. Then $G_{0}$ is a semi-direct product of the form $G_{1} \rtimes \mathbb{Z} /(m)$ where $G_{1}$ is a $p$-group and $p \nmid m$ [12, IV]. Let $J \subset G$ be the normal subgroup generated by the conjugates 
of $G_{1}$. Then the $G / J$-Galois quotient cover is a tame Galois cover of $\mathbb{P}_{k}^{1}$ branched only at $\infty$. Since the tame fundamental group of $\mathbb{A}_{k}^{1}$ is trivial, this implies that $J=G$. Based on this, Abhyankar stated the currently unproven Inertia Conjecture.

Conjecture 1.1 (Inertia Conjecture). [3, Section 16] Let $G$ be a finite quasi$p$ group. Let $G_{0}$ be a subgroup of $G$ which is an extension of a cyclic group of order prime-to-p by a p-group $G_{1}$. Then $G_{0}$ occurs as the inertia group of a ramified point of a G-Galois cover $\phi: Y \rightarrow \mathbb{P}_{k}^{1}$ branched only at $\infty$ if and only if the conjugates of $G_{1}$ generate $G$.

There is not much evidence to support the converse direction of Conjecture 1.1. For every finite quasi- $p$ group $G$, the Sylow $p$-subgroups of $G$ do occur as the inertia groups of a $G$-Galois cover of $\mathbb{P}_{k}^{1}$ branched only at $\infty$ [6]. For $p \geq 5$, Abhyankar's Inertia Conjecture is true for the quasi- $p$ groups $A_{p}$ and $\mathrm{PSL}_{2}\left(\mathbb{F}_{p}\right)$ [5, Thm. 2]. In Theorem 5.2, we prove:

Theorem 1.2. If $p \equiv 2 \bmod 3$ is an odd prime, then Abhyankar's Inertia Conjecture is true for the quasi-p group $A_{p+2}$. In other words, every subgroup $G_{0} \subset A_{p+2}$ of the form $\mathbb{Z} /(p) \rtimes \mathbb{Z} /(m)$ occurs as the inertia group of an $A_{p+2^{-}}$ Galois cover of $\mathbb{P}_{k}^{1}$ branched only at $\infty$.

Note that the values of $m$ such that $A_{p+2}$ contains a subgroup $G_{0} \simeq$ $\mathbb{Z} /(p) \rtimes \mathbb{Z} /(m)$ are exactly the divisors of $p-1$. We also give a second proof of Abhyankar's Inertia Conjecture for the group $A_{p}$ when $p \geq 5$; this proof uses the original equations of Abhyankar [2] rather than relying on the theory of semi-stable reduction.

More generally, we study the ramification filtrations of $A_{n}$-Galois covers $\phi: Y \rightarrow \mathbb{P}_{k}^{1}$ branched only at $\infty$ when $p$ is odd and $p \leq n<2 p$. This condition ensures that the order of $A_{n}$ is strictly divisible by $p$, and so the ramification filtration is determined by the order of $G_{0}$ and the upper jump $\sigma$. The upper jump is a rational number that satisfies some necessary conditions, Notation 5.5. One motivation to study the ramification filtration is that it determines the genus of $Y$.

In Theorem 4.9, we compute the order of the inertia group and the upper jump of the ramification filtration of Abhyankar's $A_{p+s}$-Galois cover $\phi_{s}$ : $Y_{s} \rightarrow \mathbb{P}_{k}^{1}$ branched only at $\infty$ when $2 \leq s<p$. This determines the genus of $Y_{s}$, which turns out to be quite small. When $\operatorname{gcd}(p-1, s+1)=1$, the inertia group is a maximal subgroup of the form $\mathbb{Z} /(p) \rtimes \mathbb{Z} /(m)$ in $A_{p+s}$. This is 
the basis of the proof of Theorem 1.2 when $s=2$. It also leads to another application, Corollary 5.6. where we use the theory of formal patching to prove:

Corollary 1.3. Suppose $2 \leq s<p$ and $\operatorname{gcd}(p-1, s+1)=1$. Then all but finitely many rational numbers $\sigma$ satisfying the obvious necessary conditions occur as the upper jump of an $A_{p+s}$-Galois cover of $\mathbb{P}_{k}^{1}$ branched only at $\infty$.

In fact, Corollary 1.3 is a strengthening of Theorem 1.2 when $s=2$. When $s>2$, the normalizer of a $p$-cycle in $A_{p+s}$ contains more than one maximal subgroup of the form $\mathbb{Z} /(p) \rtimes \mathbb{Z} /(m)$. This is because there are many elements of prime-to- $p$ order that centralize a $p$-cycle in $A_{p+s}$. Thus, when $s>2$, more equations will be needed to verify Abhyankar's Inertia Conjecture for the group $A_{p+s}$ using the strategy of this paper.

We would like to thank Irene Bouw for suggesting this approach for this project. The second author was partially supported by NSF grant 07-01303.

\section{Background}

Let $k$ be an algebraically closed field of characteristic $p \geq 3$. A curve in this paper is a smooth connected projective $k$-curve. A cover $\phi$ of the projective line branched only at $\infty$ will be called a cover of the affine line and the inertia group at a ramification point of $\phi$ above $\infty$ will be called the inertia group of $\phi$. A G-Galois cover is a Galois cover $\phi: Y \rightarrow X$ together with an isomorphism $G \simeq \operatorname{Aut}(Y / X)$; (the choice of isomorphism will not be important in this paper).

\section{$2.1 \quad$ Ramification}

Let $K$ be the function field of a $k$-curve $X$. A place $P$ of $K / k$ is the maximal ideal of a valuation ring $\mathcal{O}_{P} \subset K$. Let $\mathcal{P}_{K}$ denote the set of all such places. Let $v_{P}$ denote the normed discrete valuation on the valuation ring $\mathcal{O}_{P}$. A local parameter at $P$ is an element $\alpha \in \mathcal{O}_{P}$ such that $v_{P}(\alpha)=1$.

Consider a finite separable extension $F / K$. Let $\tilde{F}$ be the Galois closure of $F / K$ and let $G$ be the Galois group of $\tilde{F} / K$. A place $Q \in \mathcal{P}_{F}$ is said to lie over $P \in \mathcal{P}_{K}$ if $\mathcal{O}_{P}=\mathcal{O}_{Q} \cap K$ and we denote this by $Q \mid P$. For any $Q \in \mathcal{P}_{F}$ with $Q \mid P$, there is a unique integer $e(Q \mid P)$ such that $v_{Q}(x)=e(Q \mid P) v_{P}(x)$ for any $x \in K$. The integer $e(Q \mid P)$ is the ramification index of $Q \mid P$ in $F / K$. 
The extension $F / K$ is wildly ramified at $Q \mid P$ if $p$ divides $e(Q \mid P)$. When there exists a ramification point $Q$ such that $p$ divides $e(Q \mid P)$, we say that the extension is wildly ramified.

\subsection{Higher Ramification Groups}

We will need the following material from [13, Chapter 3].

Definition 2.1. For any integer $i \geq-1$ the $i$-th lower ramification group of $Q \mid P$ is

$$
G_{i}(Q \mid P)=\left\{\sigma \in G: v_{Q}(\sigma(z)-z) \geq i+1 \text { for all } z \in \mathcal{O}_{Q}\right\}
$$

We let $G_{i}$ denote $G_{i}(Q \mid P)$ when the places are clear from context.

Proposition 2.2. With the notation above, then:

1. $G_{0}$ is the inertia group of $Q \mid P$, and thus $\left|G_{0}\right|=e(Q \mid P)$, and $G_{1}$ is a p-group.

2. $G_{-1} \supseteq G_{0} \supseteq \cdots$ and $G_{h}=\{$ Id $\}$ for sufficiently large $h$.

Theorem 2.3 (Hilbert's Different Formula). The different exponent of $F / K$ at $Q \mid P$ is

$$
d(Q \mid P)=\sum_{i=0}^{\infty}\left(\left|G_{i}(Q \mid P)\right|-1\right) .
$$

Here is the Riemann-Hurwitz formula for wildly ramified extensions.

Theorem 2.4 (Riemann-Hurwitz Formula). Let $g$ (resp. $\left.g^{\prime}\right)$ be the genus of the function field $K / k$ (resp. $F / k$ ). Then

$$
2 g^{\prime}-2=[F: K](2 g-2)+\sum_{P \in \mathcal{P}_{K}} \sum_{Q \mid P} d(Q \mid P) .
$$

\subsection{Properties of Ramification Groups}

Suppose that the order of $G$ is strictly divisible by $p$. Suppose that $F / K$ is wildly ramified at $Q$. The following material about the structure of the inertia group and the higher ramification groups can be found in [12, IV]. 
Lemma 2.5. [12, IV, Cor. 4] If $F / K$ is wildly ramified at $Q \in \mathcal{P}_{F}$ with inertia group $G_{0}$ such that $p^{2} \nmid\left|G_{0}\right|$, then $G_{0}$ is a semidirect product of the form $\mathbb{Z} /(p) \rtimes \mathbb{Z} /(m)$ for some prime-to-p integer $m$.

The lower numbering on the filtration from Definition 2.1 is invariant under sub-extensions. There is a different indexing system on the filtration, whose virtue is that it is invariant under quotient extensions.

Definition 2.6. [12, IV, Section 3] The lower jump of $F / K$ of $Q \mid P$ is the largest integer $h$ such that $G_{h} \neq\{1\}$. Let $\varphi(i)=\left|G_{0}\right|^{-1} \sum_{j=1}^{i}\left|G_{j}\right|$. Define $G^{\varphi(i)}=G_{i}$. Then $\varphi(h)=h / m$. The rational number $\sigma=h / m$ is the upper jump; it is the jump in the filtration of the higher ramification groups in the upper numbering.

Let $\tau \in G_{0}$ have order $p$ and $\beta \in G_{0}$ have order $m$, so that $G_{0} \cong\langle\tau\rangle \rtimes\langle\beta\rangle$.

Lemma 2.7. [12, IV, Prop. 9] With notation as above:

1. If $\beta \in G_{0}$ has order $m$ and $h$ is the lower jump, then $\beta \tau \beta^{-1}=\beta^{h} \tau$.

2. $G_{0}$ is contained in the normalizer $N_{G}(\langle\tau\rangle)$.

\section{$2.4 \quad$ Alternating groups}

Suppose that $G$ is an alternating group $A_{n}$. Let $p \leq n<2 p$ so that $p^{2} \nmid|G|$. The following lemmas give an upper bound for the size of the inertia group.

Lemma 2.8. Let $\tau=(12 \ldots p)$. Then $N_{A_{p}}(\langle\tau\rangle)=\langle\tau\rangle \rtimes\left\langle\beta_{\circ}\right\rangle$ for some $\beta_{\circ} \in A_{p}$ with $\left|\beta_{\circ}\right|=(p-1) / 2$.

Proof. Let $n_{p}$ be the number of Sylow $p$-subgroups of $A_{p}$; then $n_{p}=\left[A_{p}\right.$ : $\left.N_{A_{p}}(\langle\tau\rangle)\right]$. There are $(p-1)$ ! different $p$-cycles in $A_{p}$, each generating a group with $p-1$ non-trivial elements. It follows that $n_{p}=(p-2)$ !. Therefore, $\left|N_{A_{p}}(\langle\tau\rangle)\right|=p(p-1) / 2$.

Clearly, $\langle\tau\rangle \subset N_{A_{p}}(\langle\tau\rangle)$; we show the existence of $\beta_{\circ}$. Let $a \in \mathbb{F}_{p}^{*}$ with $|a|=p-1$. There exists $\theta \in S_{p}$ such that $\theta \tau \theta^{-1}=\tau^{a}$. The permutation $\theta$ exists since all $p$-cycles in $S_{p}$ are in the same conjugacy class. Let $\beta_{\circ}=\theta^{2}$. Then $\beta_{\circ} \in A_{p}$ and $\beta_{\circ} \in N_{A_{p}}(\langle\tau\rangle)$. Also, for any $r$,

$$
\beta_{\circ}^{r} \tau \beta_{\circ}^{-r}=\theta^{2 r} \tau \theta^{-2 r}=\tau^{a^{2 r}} .
$$


Choosing $r=(p-1) / 2$ shows that $\beta_{\circ}^{(p-1) / 2}$ is contained in the centralizer $C_{A_{p}}(\langle\tau\rangle)=\langle\tau\rangle$, and it follows that $\beta_{\circ}^{(p-1) / 2}=1$. If $1 \leq r<(p-1) / 2$, then $\beta_{\circ}^{r} \notin C_{A_{p}}(\langle\tau\rangle)$ and thus $\beta_{\circ}^{r} \neq 1$. It follows that $\beta_{\circ}$ normalizes $\langle\tau\rangle$ in $A_{p}$ and $\beta_{\circ}$ has order $(p-1) / 2$.

Recall that $C_{S_{n}}(\langle\tau\rangle)=\langle\tau\rangle \times H$ where $H=\left\{\omega \in S_{n}: \omega\right.$ is disjoint from $\left.\tau\right\}$.

Lemma 2.9. Let $2 \leq s<p$ and let $\tau=(12 \ldots p)$. Let $H_{s} \subset S_{p+s}$ be the subgroup of permutations of the set $\{p+1, p+2, \ldots, p+s\}$. Then there exists $\theta \in S_{p}$ such that $|\theta|=p-1$ and $N_{A_{p+s}}(\langle\tau\rangle)$ is the intersection of $A_{p+s}$ with $(\langle\tau\rangle \rtimes\langle\theta\rangle) \times H_{s}$.

Proof. The permutation $\theta$ in the proof of Lemma 2.8 has order $p-1$ and normalizes $\tau$. The elements of $H_{s}$ commute with $\tau$ and $\theta$. Thus $(\langle\tau\rangle \rtimes\langle\theta\rangle) \times$ $H_{s} \subset N_{S_{p+s}}(\langle\tau\rangle)$. Performing a similar count as for Lemma 2.8, we find that the number of Sylow $p$-subgroups in $S_{p+s}$ is $(p+s) ! /(s ! p(p-1))$. Therefore $\left|N_{S_{p+s}}(\langle\tau\rangle)\right|=s ! p(p-1)$. Thus $(\langle\tau\rangle \rtimes\langle\theta\rangle) \times H_{s}=N_{S_{p+s}}(\langle\tau\rangle)$. The result follows by taking the intersection with $A_{p+s}$.

Note that the order of $N_{A_{p}}(\langle\tau\rangle)$ forces $\theta$ to be an odd permutation. Suppose $G_{0}=\langle\tau\rangle \rtimes\langle\beta\rangle$ is a subgroup of $A_{p+s}$. Then $\beta=\theta^{i} \omega$ where $\omega \in H_{s}$ and $\omega$ is an even permutation if and only if $i$ is even.

Recall that for an inertia group $G_{0}$ with $p^{2} \nmid\left|G_{0}\right|$, there is a unique lower jump $h$ which encodes information about the filtration of higher ramification groups. The following two lemmas relate the congruence class of $h$ modulo $m$ to the order of the centralizer $C_{G_{0}}(\langle\tau\rangle)$.

Lemma 2.10. Let $\pi: X \rightarrow \mathbb{P}_{k}^{1}$ be an $A_{p}$-Galois cover which is wildly ramified at a point $Q$ above $\infty$ with inertia group $G_{0}$. If $\left|G_{0}\right|=$ pm and $\pi$ has lower jump $h$ at $Q$, then $\operatorname{gcd}(h, m)=1$.

Proof. Let $\beta \in A_{p}$ be such that $G_{0}=\langle\tau\rangle \rtimes\langle\beta\rangle$. Notice that $C_{G_{0}}(\langle\tau\rangle)=\langle\tau\rangle$ since there are no elements of $A_{p}$ disjoint from $\tau$. Then $\beta^{i} \notin C_{G_{0}}(\langle\tau\rangle)$ for all $1 \leq i<m$. By Lemma 2.7(1), if $1 \leq i<m$, then $\tau \neq \beta^{i} \tau \beta^{-i}=\beta^{i h} \tau$. Notice that $\beta^{i h} \neq 1$ which implies that $m \nmid i h$ for each $1 \leq i<m$. Hence $\operatorname{gcd}(h, m)=1$.

Lemma 2.11. Let $2 \leq s<p$, and let $\phi: Y \rightarrow \mathbb{P}_{k}^{1}$ be an $A_{p+s}$-Galois cover which is wildly ramified at a point $Q$ above $\infty$ with inertia group $G_{0}$. If $\left|G_{0}\right|=$ pm and $\phi$ has lower jump $h$ at $Q$, then $C_{G_{0}}(\langle\tau\rangle) \cong \mathbb{Z} /(p) \times \mathbb{Z} /\left(m^{\prime}\right)$ where $m^{\prime}=\operatorname{gcd}(h, m)$. 
Proof. Let $\beta \in A_{p+s}$ be such that $G_{0}=\langle\tau\rangle \rtimes\langle\beta\rangle$. Let $m^{\prime}=\operatorname{gcd}(h, m)$. Then Lemma 2.7(1) implies $\beta^{m / m^{\prime}} \tau \beta^{-m / m^{\prime}}=\beta^{m \cdot h / m^{\prime}} \tau=\tau$. The last equality is true because $|\beta|=m$ and $h / m^{\prime} \in \mathbb{Z}$. It follows that $\beta^{m / m^{\prime}} \in C_{G_{0}}(\langle\tau\rangle)$, that is $\langle\tau\rangle \times\left\langle\beta^{m / m^{\prime}}\right\rangle \subset C_{G_{0}}(\langle\tau\rangle)$.

Suppose that $\alpha \in\langle\beta\rangle \cap C_{G_{0}}(\langle\tau\rangle)$. Lemma 2.7(1) implies $\tau=\alpha \tau \alpha^{-1}=$ $\alpha^{h} \tau$. It follows that $|\alpha|$ divides $h$ and $m$, so $|\alpha|$ divides $m^{\prime}$ and $\alpha \in\left\langle\beta^{m / m^{\prime}}\right\rangle$. Hence $C_{G_{0}}(\langle\tau\rangle)=\langle\tau\rangle \times\left\langle\beta^{m / m^{\prime}}\right\rangle$.

\section{Newton Polygons}

Suppose $f$ defines a degree $n$ extension $F$ of $k(x)$ that is ramified above the place $(x)$. Let $\tilde{F}$ be the splitting field of $f$ over $k(x)$. Let $Q$ be a ramified place in $\tilde{F}$ above $(x)$. Let $G_{0}$ be the inertia group of $\tilde{F} / k(x)$ at $Q$. Let $\epsilon$ be a local parameter of the valuation ring $\mathcal{O}_{Q}$. Let $v_{Q}$ denote the valuation at $Q$.

The Galois extension $\tilde{F} / k(x)$ yields a totally ramified Galois extension of complete local rings $k[[\epsilon]] / k[[x]]$. Let $f_{2} \in k[[x]][y]$ be the minimal polynomial for $\epsilon$. Let $e=e(Q \mid 0)$ be the degree of $f_{2}$. Define a polynomial $N(z) \in \hat{\mathcal{O}}_{Q}[z]$ such that

$$
\epsilon^{-e} N(z):=\epsilon^{-e} f_{2}(\epsilon(z+1))=\prod_{\omega \in G_{0}}\left(z-\left(\frac{\omega(\epsilon)-\epsilon}{\epsilon}\right)\right) .
$$

Define coefficients $b_{i} \in \hat{\mathcal{O}}_{Q}$ such that $N(z)=\sum_{i=1}^{e} b_{i} z^{i}$. The Newton polygon $\Delta$ of $N(z)$ is obtained by taking the lower convex hull of the set of points $\left\{\left(i, v_{Q}\left(b_{i}\right)\right)\right\}_{i=1}^{e}$. Since $f_{2}$ is monic, the polygon is a sequence of line segments with increasing negative slopes.

The next proposition shows that the higher ramification groups of $\tilde{F} / k(x)$ at $Q$ are determined by the slopes of $\Delta$. This is not surprising because, as in [8, Chapter 2], the Newton polygon of $N(z)$ relates the valuations of the coefficients and roots of $N(z)$ and the higher ramification groups are determined by studying the valuation of the roots of $N(z)$.

Proposition 3.1. [11, Thm. 1] Let $\left\{V_{0}, V_{1}, \ldots, V_{r}\right\}$ be the vertices of $\Delta$ and $-h_{j}$ the slope of the edge joining $V_{j-1}$ and $V_{j}$. The slopes are integral and the lower jumps in the sequence of higher ramification groups are $h_{r}<h_{r-1}<$ $\cdots<h_{1}$. 
Lemma 3.2. For $1<t<p-2$, let $f_{1, t}(y)=y^{p}-x y^{p-t}+x \in k(x)[y]$. Let $F_{t} / k(x)$ be the corresponding extension of function fields and $\tilde{F}_{t} / k(x)$ its Galois closure. Let $Q$ be a place of $\tilde{F}_{t}$ lying over 0 . Then $e(Q \mid 0)=p m$ for some integer $m$ such that $p \nmid m$. Then the Newton polygon $\Delta_{t}$ of $\tilde{F}_{t} / k(x)$ has two line segments, one having integral slope $-m(p-t) /(p-1)$ and the other having slope 0 .

Proof. Let $G$ be the Galois group of the extension $\tilde{F}_{t} / k(x)$. Notice that $G$ is contained in $S_{p}$; therefore the order of $G$ is strictly divisible by $p$. The extension is branched over $x=0$. Let $P$ and $Q$ be places lying above 0 in $F_{t}$ and $\tilde{F}_{t}$ respectively. The format of the equation $f_{1, t}$ implies that $e(P \mid 0)=p$; let $m$ be the integer such that $e(Q \mid 0)=p m$. Then $p \nmid m$ since $p^{2} \nmid|G|$. Let $G_{0}$ be the inertia group at $Q$. Let $x, \eta$, and $\epsilon$ be local parameters of $\mathcal{O}_{x}, \mathcal{O}_{P}$, and $\mathcal{O}_{Q}$ respectively. The extension $\mathcal{O}_{Q} / k[[x]]$ is totally ramified with Galois group $G_{0}$ of order pm.

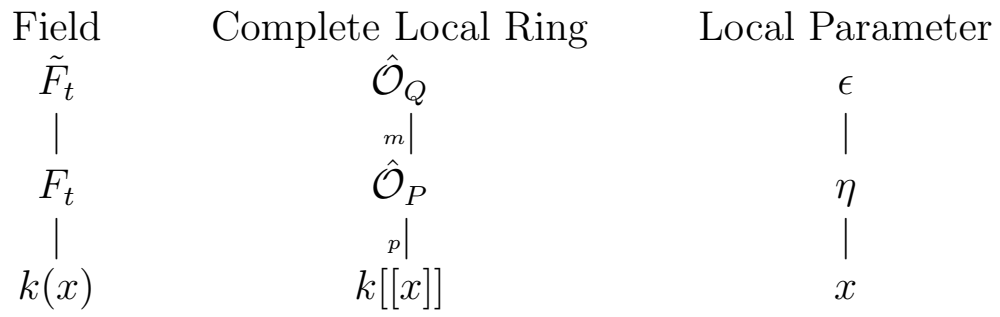

Notice that any root of $f_{1, t}$ is a local parameter at $P$ since

$$
p=v_{P}(x)=v_{P}\left(\frac{y^{p}}{y^{p-t}+1}\right)=p v_{P}(y) .
$$

Thus we can assume that $\eta$ is a root of $f_{1, t}$. Now consider $\eta$ as an element of $\hat{\mathcal{O}}_{Q}$. Then $\eta$ can be expressed as a power series in the local parameter $\epsilon$ with coefficients in $k$, that is $\eta=u \cdot \epsilon^{m}$ where $u$ is a unit of $\hat{\mathcal{O}}_{Q}$. Also $u$ is an $m$-th power in the complete local ring $\hat{\mathcal{O}}_{Q}$ so by changing the local parameter $\epsilon$ we can suppose $\eta=\epsilon^{m}$. It follows that $\epsilon$ satisfies the equation

$$
f_{2, t}(\epsilon)=\epsilon^{p m}-x \epsilon^{m(p-t)}+x=0 .
$$

The polynomial $f_{2, t}(\epsilon)$ is Eisenstein at the prime $(x)$. Now we consider

$$
N(z)=f_{2, t}(\epsilon(z+1))=\epsilon^{p m}(z+1)^{p m}-x \epsilon^{m(p-t)}(z+1)^{m(p-t)}+x .
$$


Dividing both sides of Equation 3.3 by $\epsilon^{p m}$ produces a vertical shift by $-p m$ to the Newton polygon $\Delta_{t}$. Vertical and horizontal shifts do not affect the slopes of the line segments of $\Delta_{t}$. Substituting $x=\epsilon^{p m} /\left(\epsilon^{m(p-t)}-1\right)$ and letting $d=1 /\left(\epsilon^{m(p-t)}-1\right)$, then

$$
\frac{N(z)}{\epsilon^{p m}}=(z+1)^{p m}-d \epsilon^{m(p-t)}(z+1)^{m(p-t)}+d .
$$

Notice that $N(0)=0$ so we can factor a power of $z$ from $N(z)$. The effect on $\Delta_{t}$ is a shift in the horizontal direction by -1 . This results in

$$
\frac{N(z)}{z \epsilon^{p m}}=\sum_{i=0}^{m-1}\left(\begin{array}{c}
m \\
i
\end{array}\right) z^{p(m-i)-1}+-d \epsilon^{m(p-t)} \sum_{i=0}^{m(p-t)-1}\left(\begin{array}{c}
m(p-t) \\
i
\end{array}\right) z^{m(p-t)-i-1} .
$$

Let $z^{-1} \epsilon^{-p m} N(z)=\sum_{j=0}^{p m-1} b_{j} z^{j}$. The valuation of each $b_{j}$ is greater than or equal to zero. The ramification polygon $\Delta_{t}$ is determined by calculating the valuations of the specific coefficients that determine the lower convex hull of $\Delta_{t}$ :

1. $v_{Q}\left(b_{0}\right)=v_{Q}\left(d m t \epsilon^{m(p-t)}\right)=m(p-t)$.

2. For $1 \leq j<p-1$, let $i_{j}=m(p-t)-j-1$, then

$$
v_{Q}\left(b_{j}\right)=v_{Q}\left(-d \epsilon^{m(p-t)}\left(\begin{array}{c}
m(p-t) \\
i_{j}
\end{array}\right)\right) \geq m(p-t) .
$$

3. $v_{Q}\left(b_{p-1}\right)=v_{Q}\left(m-d \epsilon^{m(p-t)}\left(\begin{array}{c}m(p-t) \\ m(p-t)-p)\end{array}\right)\right)=0$.

4. $v_{Q}\left(b_{p m-1}\right)=v_{Q}(1)=0$.

The vertices of $\Delta_{t}$ are thus $(0, m(p-t)),(p-1,0)$, and $(p m-1,0)$.

Lemma 3.3. For $2 \leq s<p$, let $g_{s}(y)=y^{p+s}-x y^{s}+1 \in k(x)[y]$. Let $L_{s} / k(x)$ be the corresponding extension of function fields and $\tilde{L}_{s} / k(x)$ its Galois closure. Let $Q$ be a place of $\tilde{L}_{s}$ lying over $\infty$. Then e $(Q \mid \infty)=$ pm for some integer $m$ such that $p \nmid m$ and the Newton polygon $\Delta_{s}^{\prime}$ of $\tilde{L}_{s} / k(x)$ has two line segments, one having integral slope $-m(p+s) /(p-1)$ and the other having slope 0. 
Proof. Let $G$ be the Galois group of the extension $\tilde{L}_{s} / k(x)$. Notice that $G$ is contained in $S_{p+s}$; therefore the order of $G$ is strictly divisible by $p$. The extension is branched over $\infty$. Let $P_{(\infty, 0)}$ and $P_{(\infty, \infty)}$ be the two places of $L_{s}$ lying above $\infty$. The format of the equation $g_{s}$ implies that $P_{(\infty, 0)}$ and $P_{(\infty, \infty)}$ have ramification indices $p$ and $s$ respectively, see e.g., Lemma 4.8. Let $Q$ be a place of $\tilde{L}_{s}$ lying above $P_{(\infty, 0)}$. Let $m$ be the integer $e(Q \mid \infty) / p$. Let $G_{0}$ be the inertia group at $Q$. Let $x^{-1}, \eta$, and $\epsilon$ be local parameters of $\mathcal{O}_{x^{-1}}, \mathcal{O}_{P_{(\infty, 0)}}$, and $\mathcal{O}_{Q}$ respectively.

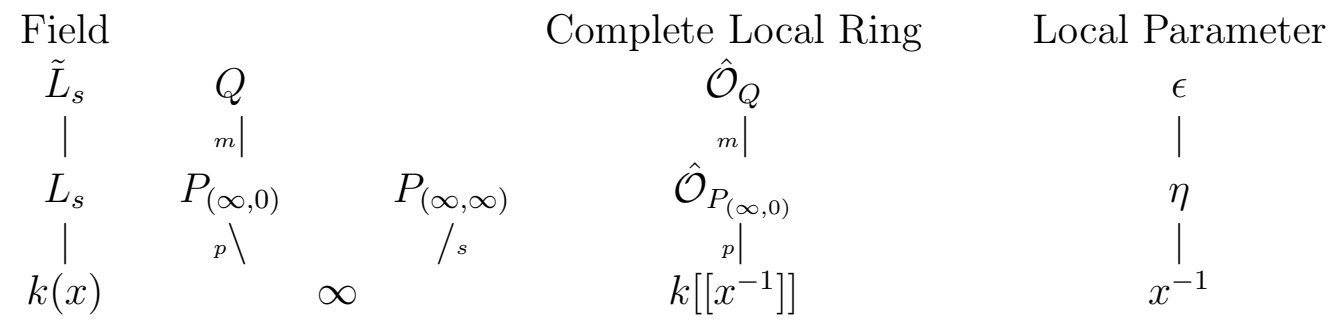

Then $\hat{\mathcal{O}}_{Q} / k\left[\left[x^{-1}\right]\right]$ is a totally ramified Galois extension with Galois group $G_{0}$ of order $p m$. By the same reasoning as for Lemma 3.2, there exists a local parameter $\epsilon$ of $\hat{\mathcal{O}}_{Q}$ that satisfies $\epsilon^{m}=\eta$. Therefore $\epsilon$ satisfies the irreducible equation

$$
g_{2, s}(\epsilon)=\epsilon^{m(p+s)}-x \epsilon^{m s}+1=0 .
$$

We calculate the ramification polygon $\Delta_{s}^{\prime}$ by considering

$$
N(z)=g_{2, s}(\epsilon(z+1))=\epsilon^{m(p+s)}(z+1)^{m(p+s)}-x \epsilon^{m s}(z+1)^{m s}+1 .
$$

Since $N(0)=0$, it follows that

$$
\frac{N(z)}{z \epsilon^{m(p+s)}}=(z+1)^{m s} \sum_{i=0}^{m-1}\left(\begin{array}{c}
m \\
i
\end{array}\right) z^{p(m-i)-1}-\left(1+\epsilon^{-m(p-s)}\right) \sum_{i=0}^{m s-1}\left(\begin{array}{c}
m s \\
i
\end{array}\right) z^{m s-1-i} .
$$

Let $z^{-1} \epsilon^{-m(p+s)} N(z)=\sum_{j=1}^{m(p+s)-1} b_{j} z^{j}$. The valuation of each $b_{j}$ is nonnegative. The ramification polygon $\Delta_{s}^{\prime}$ is determined when we calculate the valuations of the specific coefficients that determine the lower convex hull of $\Delta_{s}^{\prime}$.

1. $v_{Q}\left(b_{0}\right)=m(p+s)$.

2. $v_{Q}\left(b_{j}\right) \geq m(p+s)$ for $1 \leq j<p-1$. 
3. $v_{Q}\left(b_{p-1}\right)=0$.

4. $v_{Q}\left(b_{m(p+s)-1}\right)=0$.

The vertices of $\Delta_{s}^{\prime}$ are thus $(0, m(p-s)),(p-1,0)$, and $(m(p+s)-1,0)$.

\section{$4 A_{n}$-Galois covers of the affine line}

Suppose $\pi: X \rightarrow \mathbb{P}_{k}^{1}$ is an $A_{n}$-Galois cover branched only at $\infty$. The cover is wildly ramified at each point $Q \in X$ above $\infty$. The complexity of the wild ramification is directly related to the power of $p$ that divides the ramification index $e(Q \mid \infty)$. For this reason, we concentrate on Galois groups $A_{n}$ such that the order of $A_{n}$ is strictly divisible by $p$. We use some equations of Abhyankar to study $A_{n}$-Galois covers when $p$ is odd and $p \leq n<2 p$. The goal is to determine the inertia groups and upper jumps that occur for $A_{n^{-}}$ Galois covers $\pi: X \rightarrow \mathbb{P}_{k}^{1}$ branched only at $\infty$. This ramification data also determines the genus of the curve $X$.

\subsection{Two useful lemmas}

The following is a version of Abhyankar's Lemma which will be needed to construct a $G$-Galois cover of $\mathbb{P}_{k}^{1}$ branched only at $\infty$ from a $G$-Galois cover of $\mathbb{P}_{k}^{1}$ branched at 0 and $\infty$.

Lemma 4.1 (Refined Abhyankar's Lemma). Let $m, r_{1}$, and $r_{2}$ be primeto-p integers. Suppose $\pi: X \rightarrow \mathbb{P}_{k}^{1}$ is a G-Galois cover with branch locus $\{0, \infty\}$. Suppose $\pi$ has ramification index $r_{1}$ above 0 and inertia group $G_{0} \cong$ $\mathbb{Z} /(p) \rtimes \mathbb{Z} /(m)$ above $\infty$ with lower jump $h$. Let $\psi: \mathbb{P}_{k}^{1} \rightarrow \mathbb{P}_{k}^{1}$ be an $r_{2}$-cyclic cover with branch locus $\{0, \infty\}$. Assume that $\pi$ and $\psi$ are linearly disjoint.

Then the pullback $\pi^{\prime}=\psi^{*} \pi$ is a G-Galois cover $\pi^{\prime}: X^{\prime} \rightarrow \mathbb{P}_{k}^{1}$ with branch locus contained in $\{0, \infty\}$, with ramification index $r_{1} / \operatorname{gcd}\left(r_{1}, r_{2}\right)$ above 0 , with inertia group $G_{0}^{\prime} \subset G_{0}$ of order $\mathrm{pm} / \operatorname{gcd}\left(m, r_{2}\right)$ above $\infty$ and with lower jump $h r_{2} / \operatorname{gcd}\left(m, r_{2}\right)$. If $\sigma$ and $\sigma^{\prime}$ are the upper jumps of $\pi$ and $\pi^{\prime}$ respectively, then $\sigma^{\prime}=r_{2} \sigma$.

Proof. Consider the fibre product:

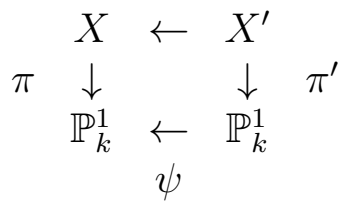


All the claims follow from the classical version of Abhyankar's Lemma [7, Lemma X.3.6] except for the information about the lower and upper jumps of $\pi^{\prime}$. Consider the composition $\psi \pi^{\prime}$ which has ramification index $p m r_{2} / \operatorname{gcd}\left(m, r_{2}\right)$ above $\infty$. Since upper jumps are invariant under quotients, the upper jump of $\psi \pi^{\prime}$ equals $\sigma$. Thus the lower jump of $\psi \pi^{\prime}$ equals $\sigma m r_{2} / \operatorname{gcd}\left(m, r_{2}\right)=$ $h r_{2} / \operatorname{gcd}\left(m, r_{2}\right)$ by Definition [2.6. This equals the lower jump of $\pi^{\prime}$ since lower jumps are invariant for subcovers and the claim about the upper jump of $\pi^{\prime}$ follows from Definition 2.6.

The following lemma is useful to compare ramification information about a cover and its Galois closure. Let $S_{n}^{1}:=\operatorname{Stab}_{S_{n}}(1)$.

Lemma 4.2. If $\rho: Z \rightarrow W$ is a cover with Galois closure $\pi: X \rightarrow W$, then the branch locus of $\rho$ and of $\pi$ are the same.

Proof. The branch locus of $\rho$ is contained in the branch locus of $\pi$ since ramification indices are multiplicative. Assume that $b$ is in the branch locus of $\pi$ but not in the branch locus of $\rho$. We will show that this is impossible. The Galois group $H$ of $\pi$ is a transitive subgroup of $S_{n}$, where $n$ is the degree of $\rho$. The Galois group $H^{\prime}$ of $X \rightarrow Z$ is a subgroup of $H$ with index $n$. After identifying $H$ with a subgroup of $S_{n}$, we can assume without loss of generality that $H^{\prime} \subset S_{n}^{1}$. Let $Q \in X$ be a ramification point lying above $b$ with inertia group $G_{0}$. Conjugating $G_{0}$ by an element $\omega \in H$ results in an inertia group at some point of $X$ above $b$. Since $b$ is not a branch point of $\rho$, we have that $\omega G_{0} \omega^{-1} \subset S_{n}^{1}$ for all $\omega \in H$. This is impossible since $H$ is transitive on the set $\{1,2, \ldots, n\}$. Therefore the branch loci must be the same.

\section{2 $A_{p}$-Galois covers of the affine line}

Let $p \geq 5$. In this section, we find $A_{p}$-Galois covers $\pi: X \rightarrow \mathbb{P}_{k}^{1}$ branched only at $\infty$ with a small upper jump.

Notation 4.3. Let $t$ be an integer with $1<t<p-2$ and let $f_{t}=y^{p}-y^{t}+x$. Consider the curve $Z_{t}$ with function field $F_{t}:=k(x)[y] /\left(f_{t}\right)$. Let $\pi_{t}: X_{t} \rightarrow \mathbb{P}_{k}^{1}$ be the Galois closure of $\rho_{t}: Z_{t} \rightarrow \mathbb{P}_{k}^{1}$; the function field of $X_{t}$ is the Galois closure $\tilde{F}_{t}$ of $F_{t} / k(x)$. Let $\zeta$ be a $(p-t)$ th root of unity.

Abhyankar proved that the Galois group of $\pi_{t}$ is $A_{p}$ when $t$ is odd and $S_{p}$ when $t$ is even [2, Section 20]. For the proof, he showed that the Galois group is doubly transitive on the set $\{1,2, \ldots, p\}$ and contains a certain cycle 
type. We now study the ramification of the cover $\pi_{t}$. The following result can be found in [2, Section 20].

Lemma 4.4. The cover $\pi_{t}: X_{t} \rightarrow \mathbb{P}_{k}^{1}$ has one ramified point above $x=0$ with ramification index $t$ and is unramified above all other points of $\mathbb{A}_{k}^{1}$.

Proof. When $x=0$ in the equation $f_{t}$, then $y=0$ or $y=\zeta^{i}$ for some $1 \leq i \leq p-t$. There are $p-t+1$ points in the fibre of $\rho$ above the point $x=0$ which we denote by $P_{(0,0)}$ and $P_{(0, \zeta)}, \ldots, P_{(0,1)}$. Since $p-t+1<p$, then $x=0$ is a branch point of $\rho$.

The value $y=0$ is the only solution to $\partial f / \partial y=0$. Therefore $P_{(0,0)}$ is the only ramification point above $\mathbb{A}_{k}^{1}$. The Galois group $H$ of $\pi_{t}$ is either $S_{p}$ or $A_{p}$. Thus Lemma 4.2 implies that $\pi_{t}$ is unramified above all points of $\mathbb{A}_{k}^{1}$ except $x=0$.

Because $p=\sum_{P \mid 0} e(P \mid 0)$, it follows that $P_{(0,0)}$ has ramification index $t$. Let $Q \in X_{t}$ be a point lying above $P_{(0,0)}$. It remains to show that $e\left(Q \mid P_{(0,0)}\right)=$ 1 .

Let $H^{\prime}$ be the Galois group of $X_{t} \rightarrow Z_{t}$. Without loss of generality we can suppose that $H^{\prime} \subset S_{p}^{1}$. Since $p \nmid\left|H^{\prime}\right|$, Lemma 2.5 implies that $G_{0}(Q \mid 0)$ is a cyclic group of order $t \cdot c$ for some prime-to- $p$ integer $c$.

Assume $c \neq 1$. If $\omega$ is a generator for $G_{0}(Q \mid 0)$; then $\omega \notin S_{p}^{1}$ since $P_{(0,0)}$ is ramified over 0 . Then $G_{0}\left(Q \mid P_{(0,0)}\right)=\left\langle\omega^{t}\right\rangle \subset S_{p}^{1}$. By the assumption on $c$, the automorphism $\omega^{t}$ is not the identity. Since $H$ is transitive on $\{1,2, \ldots, p\}$, there exists $\gamma \in H$ such that $\gamma \phi^{t} \gamma^{-1} \notin S_{p}^{1}$.

There exists a point $\tilde{Q}$ in the fiber of $X_{t}$ above 0 such that $G_{0}(\tilde{Q} \mid 0)=$ $\left\langle\gamma^{-1} \phi \gamma\right\rangle$. Since $\gamma \notin S_{p}^{1}$, the point $\tilde{Q}$ is in the fibre of $\pi_{t}$ over 0 but not in the fibre above $P_{(0,0)}$. Furthermore, $\gamma \phi^{t} \gamma^{-1}=\left(\gamma \phi \gamma^{-1}\right)^{t} \in G_{0}(\tilde{Q} \mid 0)$. Hence $G_{0}(\tilde{Q} \mid 0) \not \subset S_{p}^{1}$. Therefore, for some $i$, the extension $P_{\left(0, \zeta^{i}\right)} \mid 0$ is ramified. This gives a contradiction so the assumption that $c \neq 1$ is false.

Lemma 2.5 implies that the inertia group $G_{0}$ at a point of $X_{t}$ over $\infty$ is of the form $\mathbb{Z} /(p) \rtimes \mathbb{Z} /(m)$ where $p \nmid m$. To determine the upper jump $\sigma$ of $\pi_{t}$ over $\infty$, we use the equation $f_{t}$ to understand the ramification that occurs in the quotient map $\rho_{t}: Z_{t} \rightarrow \mathbb{P}_{k}^{1}$.

Lemma 4.5. The cover $\pi_{t}: X_{t} \rightarrow \mathbb{P}_{k}^{1}$ has ramification index $p(p-1) / \operatorname{gcd}(p-$ $1, t-1)$ and upper jump $\sigma=(p-t) /(p-1)$ above $\infty$. 
Proof. Let $P_{\infty}$ be a point of $Z_{t}$ that lies above $\infty$. Then

$$
-e\left(P_{\infty} \mid \infty\right)=v_{P_{\infty}}(x)=v_{P_{\infty}}\left(y^{p}-y^{t}\right)=p v_{P_{\infty}}(y) .
$$

Therefore $p \mid e\left(P_{\infty} \mid \infty\right)$ and it follows that $\rho$ is totally ramified at $P_{\infty}$.

Consider the change of variables $x \mapsto 1 / x$ and $y \mapsto 1 / y$. Understanding the ramification over $\infty$ is equivalent to understanding the ramification over $x=0$ of the cover with equation $f_{1, t}=y^{p}-x y^{p-t}+x$. By Lemma 3.2, the absolute value of the non-zero slope of the ramification polygon $\Delta_{t}$ of $\tilde{F}_{t} / k(x)$ is $m(p-t) /(p-1)$; this is the lower jump by Lemma 3.1. By Definition 2.6. the upper jump is $\sigma=(p-t) /(p-1)$.

By Lemma 2.10, $h$ and $m$ are co-prime, therefore $h=(p-t) / \operatorname{gcd}(p-$ $1, t-1)$ and $m=(p-1) / \operatorname{gcd}(p-1, t-1)$ and $\left|G_{0}\right|=p m$.

Theorem 4.6. For $1<t<(p-2)$, let $m_{t}=(p-1) / \operatorname{gcd}(p-1, t(t-1))$. Then there exists an $A_{p}$-Galois cover $\pi_{t}^{\prime}: X_{t}^{\prime} \rightarrow \mathbb{P}_{k}^{1}$ branched only at $\infty$ with ramification index $\mathrm{pm}_{t}$ and upper jump $\sigma_{t}^{\prime}=t(p-t) /(p-1)$. The genus of $X_{t}^{\prime}$ is $1+\left|A_{p}\right|\left(t(p-t)-p-1 / m_{t}\right) / 2 p$.

Proof. Let $d_{1}=\operatorname{gcd}(p-1, t-1)$ and let $m=(p-1) / d_{1}$. Consider the Galois cover $\pi_{t}: X_{t} \rightarrow \mathbb{P}_{k}^{1}$ from Notation 4.3. Lemma 4.4 states that $\pi_{t}$ has ramification index $t$ above 0 and is unramified above $\mathbb{A}_{k}^{1}-\{0\}$. Lemma 4.5 states that the inertia group $G_{0}$ above $\infty$ has order $p m$ and upper jump $\sigma_{t}=(p-t) /(p-1)$.

If $t$ is odd, then $\pi_{t}$ has Galois group $A_{p}$. Let $m^{*}=\operatorname{gcd}(m, t)$. Since $A_{p}$ is simple, the cover $\pi_{t}$ is linearly disjoint from the $t$-cyclic cover $\psi: \mathbb{P}_{k}^{1} \rightarrow \mathbb{P}_{k}^{1}$ with equation $z^{t}=x$. Applying Lemma 4.1, the pullback $\pi_{t}^{\prime}=\psi^{*} \pi_{t}$ is a cover $\pi_{t}^{\prime}: X_{t}^{\prime} \rightarrow \mathbb{P}_{k}^{1}$ with Galois group $A_{p}$. The map $\pi_{t}^{\prime}$ is branched only at $\infty$ with inertia group $G_{0}^{\prime}$ of order $p m / m^{*}$ and upper jump $\sigma_{t}^{\prime}=t(p-t) /(p-1)$. Notice that $d_{1} m^{*}=\operatorname{gcd}(p-1, t(t-1))$, so the inertia group has order $p m / m^{*}=$ $p(p-1) / \operatorname{gcd}(p-1, t(t-1))$.

If $t$ is even, then $\pi_{t}$ has Galois group $S_{p}$. Let $Y_{t}$ be the smooth projective curve corresponding to the fixed field $\tilde{F}_{t}^{A_{p}}$. Let $\mu_{t}: X_{t} \rightarrow Y_{t}$ be the subcover with Galois group $A_{p}$.

The branch locus of the degree 2 quotient cover $Y_{t} \rightarrow \mathbb{P}_{k}^{1}$ is contained in $\{0, \infty\}$. The ramification index must be 2 over both 0 and $\infty$. By the Riemann-Hurwitz formula, $Y_{t}$ has genus 0 . Therefore $\mu_{t}: X_{t} \rightarrow \mathbb{P}_{k}^{1}$ is an $A_{p}$-Galois cover of the projective line. 
Let $P_{0}$ (resp. $\left.P_{\infty}\right)$ be the point of $Y_{t}$ above 0 (resp. $\infty$ ). Since ramification indices are multiplicative, $\mu_{t}$ has ramification index $t / 2$ over $P_{0}$ and $\left|G_{0}\right| / 2$ over $P_{\infty}$. It can be seen that $\left|G_{0}\right| / 2=p m / 2$ is an integer from Lemma 4.6 since $t$ is even. The lower jump of $\mu_{t}$ is the same as the lower jump of $\pi_{t}$ since lower jumps are invariant under subextensions. Therefore the upper jump of $\mu_{t}$ is $2 \sigma$.

The cover $\mu_{t}$ is linearly disjoint from the $t / 2$-cyclic cover $\psi: \mathbb{P}_{k}^{1} \rightarrow \mathbb{P}_{k}^{1}$ with equation $z^{t / 2}=x$. Let $\bar{m}=\operatorname{gcd}(m / 2, t / 2)$. Applying Lemma 4.1, the pullback $\pi_{t}^{\prime}=\psi^{*} \mu_{t}$ is an $A_{p}$-Galois cover $\pi_{t}^{\prime}: X_{t}^{\prime} \rightarrow \mathbb{P}_{k}^{1}$. The map $\pi_{t}^{\prime}$ is branched only at $\infty$ where it has inertia group $G_{0}^{\prime}$ of order $p m /(2 \bar{m})$ and upper jump $\sigma_{t}^{\prime}=t(p-t) /(p-1)$. Notice that $p m / 2 \bar{m}=p m / m^{*}$, so $\left|G_{0}^{\prime}\right|=p(p-1) / \operatorname{gcd}(p-1, t(t-1))$.

The genus calculation is immediate from the Riemann-Hurwitz formula, Theorem 2.4,

The smallest genus for an $A_{p}$-Galois cover obtained using the method of Theorem 4.6 is

$$
g=1+\left|A_{p}\right|\left(p^{2}-5 p+2\right) / 2 p(p-1) .
$$

This occurs when $t=2$ or $t=p-2$ and the upper jump is $\sigma=2(p-2) /(p-1)$. To see this, consider the derivative $d \sigma / d t=(p-2 t)(p-1)$. Since this value of $\sigma$ is less than 2, it is possible that this is the smallest genus that occurs among all $A_{p}$-Galois covers of the affine line. We find $A_{p}$-Galois covers with slightly larger upper jumps in Section 5.4.

\section{3 $A_{p+s}$-Galois covers of the affine line}

In this section, we find $A_{n}$-Galois covers of the projective line branched only at $\infty$ with small upper jump when $p$ is odd and $p<n<2 p$.

Notation 4.7. Let $s$ be an integer with $2 \leq s<p$. Consider the group $A_{p+s}$ of even permutations on $p+s$ elements and the subgroup $H_{s} \subset S_{p+s}$ of permutations on $\{p+1, p+2, \ldots, p+s\}$. Let $g_{s}=y^{p+s}-x y^{s}+1$. Consider the curve $Z_{s}^{\prime}$ with function field $L_{s}:=k(x)[y] /\left(g_{s}\right)$. Let $\phi_{s}: Y_{s} \rightarrow \mathbb{P}_{k}^{1}$ be the Galois closure of $\rho_{s}^{\prime}: Z_{s}^{\prime} \rightarrow \mathbb{P}_{k}^{1}$; the function field of $Y_{s}$ is the Galois closure $\tilde{L}_{s}$ of $L_{s} / k(x)$.

Abhyankar proved that the Galois group of $\phi_{s}$ is $A_{p+s}$ except when $p=7$ and $s=2$ [2, Section 11]. The following result can be found in [2, Section 21]. 
Lemma 4.8. The cover $\rho_{s}^{\prime}$ is branched only at $\infty$. The fibre over $\infty$ consists of two points $P_{(\infty, 0)}$ and $P_{(\infty, \infty)}$ which have ramification indices $p$ and $s$ respectively.

Proof. There are no simultaneous solutions to the equations $g_{s}=0$ and $\partial g_{s} / \partial y=0$. Therefore the cover $\rho_{s}^{\prime}$ is not branched over any points of $\mathbb{A}_{k}^{1}$. Since the tame fundamental group of $\mathbb{A}_{k}^{1}$ is trivial, $\rho_{s}^{\prime}$ must be wildly ramified above $\infty$. The fibre of $Z_{s}^{\prime}$ over $\infty$ consists of two points $P_{(\infty, 0)}$ and $P_{(\infty, \infty)}$. The first point can be seen by applying the change of variables $x \mapsto 1 / x$ to $g_{s}$. This produces the equation $x y^{p+s}-y^{s}+x$. Taking the partial derivative with respect to $y$ yields the point $P_{(\infty, 0)}$. The second point can be seen by applying the change of variables $y \mapsto 1 / y$ to $x y^{p+s}-y^{s}+x$ resulting in the equation $x-y^{p}+x y^{p+s}$. Taking the partial derivative with respect to $y$ yields the point $P_{(\infty, \infty)}$. To show that $e\left(P_{(\infty, 0)} \mid \infty\right)=p$ and $e\left(P_{(\infty, \infty)} \mid \infty\right)=s$, let $P$ be either $P_{(\infty, 0)}$ or $P_{(\infty, \infty)}$ and consider the valuation $v_{P}$. The result follows since

$$
-e(P \mid \infty)=v_{P}(x)=v\left(y^{p}+y^{-s}\right)=\min \left\{p v_{P}(y),-s v_{P}(y)\right\}
$$

Theorem 4.9. Let $2 \leq s<p$. If $p=7$, assume $s \neq 2$. Let $m_{s}=(p-$ $1) s / \operatorname{gcd}(p-1, s(s+1))$. Then there exists an $A_{p+s}$-Galois cover $\phi_{s}: Y_{s} \rightarrow \mathbb{P}_{k}^{1}$ branched only at $\infty$ with inertia group $G_{0}$ of order $p_{s}$ and upper jump $\sigma_{s}=(p+s) /(p-1)$. The genus of $Y_{s}$ is $1+\left|A_{p+s}\right|\left(s-1 / m_{s}\right) / 2 p$.

In [4, Cor. 2.2], the author proves that the genus of $Y_{s}$ in Theorem 4.9 is the smallest genus that occurs among all $A_{p+s}$-Galois covers of the affine line.

Proof. Consider the cover $\phi_{s}: Y_{s} \rightarrow \mathbb{P}_{k}^{1}$ defined in Notation 4.7. Abhyankar proved that $\phi_{s}$ has Galois group $A_{p+s}[2$, Section 11]. By Lemmas 4.2 and 4.8. $\infty$ is the only branch point of $\phi_{s}$. Let $Q$ be a point of $Y_{s}$ lying above $\infty$. The cover $\phi_{s}$ is wildly ramified at $Q$ with $p^{2} \nmid e(Q \mid \infty)$. By Lemma 2.5, the inertia group $G_{0}$ at $Q$ is of the form $\mathbb{Z} /(p) \rtimes \mathbb{Z} /(m)$ for some prime-to- $p$ integer $m$.

Let $h$ be the lower jump of $\phi_{s}$ at $Q$. The Newton polygon of $\phi_{s}$ is the same as the Newton polygon $\Delta_{s}^{\prime}$ calculated in Lemma 3.3. Therefore, $h=$ $m(p+s) /(p-1)$, because this is the negative of the slope of the line segment of $\Delta_{s}^{\prime}$. By Definition 2.6, the upper jump is $\sigma_{s}=(p+s) /(p-1)$. 
Write $m=m^{\prime} m^{\prime \prime}$ where $m^{\prime}$ is the order of the prime-to- $p$ center of $G_{0}$. Lemma 2.7(1) implies that $m^{\prime}=\operatorname{gcd}(h, m)$. Since $h / m=\sigma_{s}=(p+s) /(p-1)$, it follows that $m^{\prime \prime}=(p-1) / \operatorname{gcd}(p-1, s+1)$.

Without loss of generality, we can suppose that $\tau=(12 \ldots p) \in G_{0}$. By Lemma 2.9, $G_{0}=\langle\tau\rangle \rtimes\langle\beta\rangle$ for some $\beta$ of the form $\beta=\theta^{i} \omega$. Recall that $\theta \in S_{p}$ acts faithfully by conjugation on $\tau$ and $\omega \in H_{s}$ commutes with $\tau$. The inertia group $G_{0}$ acts transitively on $\{p+1, p+2, \ldots, p+s\}$ by Lemma 4.8. Thus $\omega$ is a cycle of length $s$.

The order of $\beta$ is $m$, the order of $\theta^{i}$ is $m^{\prime \prime}$, and the order of $\beta$ is $s$. Thus $m=\operatorname{lcm}\left(m^{\prime \prime}, s\right)$. It follows that $m^{\prime}=s / \operatorname{gcd}(p-1, s)$ and $m=(p-1) s / \operatorname{gcd}(p-$ $1, s(s+1))$. The genus calculation is immediate from the Riemann-Hurwitz formula, Theorem 2.4.

\section{Applications}

\subsection{Support for the Inertia Conjecture}

In this section, we first give a new proof of Abhyankar's Inertia Conjecture for the group $A_{p}$; this proof does not use the theory of semi-stable reduction. Then we prove Abhyankar's Inertia Conjecture for the group $A_{p+2}$ for an odd prime $p \equiv 2 \bmod 3$.

Corollary 5.1. [5, Cor. 3.1.5] Let $p \geq 5$. Abhyankar's Inertia Conjecture is true for the alternating group $A_{p}$. In other words, every subgroup $G_{0} \subset A_{p}$ of the form $\mathbb{Z} /(p) \rtimes \mathbb{Z} /(m)$ can be realized as the inertia group of an $A_{p}$-Galois cover of $\mathbb{P}_{k}^{1}$ branched only at $\infty$.

Proof. Suppose $G_{0} \subset A_{p}$ satisfies the conditions of Conjecture 1.1. Since $p^{2} \nmid\left|A_{p}\right|$, then $G_{0} \simeq \mathbb{Z} /(p) \rtimes \mathbb{Z} /(m)$ for some prime-to- $p$ integer $m$. Thus the second claim implies the first.

Consider a subgroup $G_{0} \subset A_{p}$ of the form $\mathbb{Z} /(p) \rtimes \mathbb{Z} /(m)$. The goal is to show that $G_{0}$ is the inertia group of an $A_{p}$-Galois cover of the affine line. Without loss of generality, we can suppose that $\tau=(12 \ldots p) \in G_{0}$. By Lemma 2.7(2), $G_{0} \subset N_{A_{p}}(\langle\tau\rangle)$. Lemma 2.8 implies that $N_{A_{p}} \simeq \mathbb{Z} /(p) \rtimes$ $\mathbb{Z} /((p-1) / 2)$.

It thus suffices to prove, for every $m \mid(p-1) / 2$, that there exists an $A_{p}$-Galois cover of the affine line, with an inertia group of order $\mathrm{pm}$. Letting $t=2$, Theorem 4.6 shows the existence of such a cover $\pi_{2}$ with an inertia 
group of order $p(p-1) / 2$. Since $A_{p}$ is simple, $\pi_{2}$ is linearly disjoint from the degree $r_{2}$ cyclic cover of $\mathbb{P}_{k}^{1}$ which is branched at 0 and $\infty$. The proof then follows by Lemma 4.1, taking $r_{2}=(p-1) / 2 m$.

Corollary 5.2. If $p \equiv 2 \bmod 3$ is an odd prime, then Abhyankar's Inertia Conjecture is true for $G=A_{p+2}$. In other words, every subgroup $G_{0} \subset A_{p+2}$ of the form $\mathbb{Z} /(p) \rtimes \mathbb{Z} /(m)$ can be realized as the inertia group of an $A_{p+2}$ Galois cover of $\mathbb{P}_{k}^{1}$ branched only at $\infty$.

Proof. Suppose $G_{0} \subset A_{p+2}$ satisfies the conditions of Conjecture 1.1. Since $p^{2} \nmid\left|A_{p+2}\right|$, then $G_{0} \simeq \mathbb{Z} /(p) \rtimes \mathbb{Z} /(m)$ for some prime-to- $p$ integer $m$. Thus the second claim implies the first.

Consider a subgroup $G_{0} \subset A_{p+2}$ of the form $\mathbb{Z} /(p) \rtimes \mathbb{Z} /(m)$. The goal is to show that $G_{0}$ is the inertia group of an $A_{p+2}$-Galois cover of the affine line. Without loss of generality, we can suppose that $\tau=(12 \ldots p) \in G_{0}$. By Lemma 2.7(2), $G_{0} \subset N_{A_{p+2}}(\langle\tau\rangle)$. By Lemma 2.9, $N_{A_{p+2}}(\langle\tau\rangle)=\langle\tau\rangle \rtimes\langle\beta\rangle$ where $\beta=\theta(p+1 p+2)$. Recall that $\theta$ is an odd permutation of order $p-1$ defined in the proof of Lemma 2.8.

It thus suffices to prove, for every $m \mid(p-1)$, that there exists an $A_{p+2^{-}}$ Galois cover of the affine line, with an inertia group of order $p m$. Letting $s=2$, Theorem 4.9 shows the existence of such a cover $\phi_{2}$ with an upper jump $\sigma_{2}=(p+2) /(p-1)$. Since $p \equiv 2 \bmod 3$, the upper jump $\sigma_{2}$ is written in lowest terms and thus $m=p-1$. Since $A_{p+2}$ is simple, $\phi_{2}$ is linearly disjoint from a degree $r_{2}$ cyclic cover of $\mathbb{P}_{k}^{1}$ which is branched at 0 and $\infty$. The proof then follows by Lemma 4.1, taking $r_{2}=(p-1) / m$.

When $s>2$, more equations are needed to prove Abhyankar's Conjecture for $A_{p+s}$ because the normalizer $N_{A_{p+s}}(\langle\tau\rangle)$ contains more that one maximal subgroup of the form $\mathbb{Z} /(p) \rtimes \mathbb{Z} /(m)$.

\subsection{Formal Patching Results}

Suppose $\pi: X \rightarrow \mathbb{P}_{k}^{1}$ is a $G$-Galois cover which is wildly ramified above $\infty$ with last upper jump $\sigma$. Using the theory of formal patching, it is possible

to produce a different $G$-Galois cover with the same branch locus, but with a larger upper jump above $\infty$. The formal patching proof is non-constructive and we do not describe it in this paper. Here are the results that we will use: the first allows us to change the congruence value of the lower jump modulo $m$ and the second allows us to increase the lower jump by a multiple of $m$. 
Lemma 5.3. [5, Prop. 3.1.1] Suppose $\pi: X \rightarrow \mathbb{P}_{k}^{1}$ is a G-Galois cover branched only at $\infty$ with inertia group $G_{0} \cong \mathbb{Z} /(p) \rtimes \mathbb{Z} /(m)$ with $p \nmid m$ and with lower jump $h$. For each $d \in \mathbb{N}$ such that $1 \leq d \leq m$, let $m_{d}=$ $m / \operatorname{gcd}(m, d)$ and $h_{d}=d h / \operatorname{gcd}(m, d)$. Let $G_{0}^{d} \subset G_{0}$ be the subgroup of order $p m_{d}$. Then there exists a G-Galois cover $\pi^{\prime}: X^{\prime} \rightarrow \mathbb{P}_{k}^{1}$ branched only at $\infty$ with inertia group $G_{0}^{d}$ and lower jump $h_{d}$. If $\sigma$ and $\sigma^{\prime}$ are the upper jumps of $\pi$ and $\pi^{\prime}$ respectively, then $\sigma^{\prime}=d \sigma$.

Theorem 5.4. [9, Special case of Theorem 2.3.1] Let $\pi: X \rightarrow \mathbb{P}_{k}^{1}$ be a $G$-Galois cover branched only at $\infty$ with inertia group $\mathbb{Z} /(p) \rtimes \mathbb{Z} /(m)$ and upper jump $\sigma=h / m$. Then for $i \in \mathbb{N}$ with $\operatorname{gcd}(h+i m, p)=1$, there exists a G-Galois cover branched only at $\infty$ with the same inertia group and upper jump $\sigma^{\prime}=\sigma+i$.

\subsection{Realizing almost all upper jumps for $A_{p+s}$-Galois covers}

Here are the necessary conditions on the upper jump of an $A_{p+s}$-Galois cover of the affine line.

Notation 5.5. Let $2 \leq s<p$. Suppose $\phi: Y \rightarrow \mathbb{P}_{k}^{1}$ is an $A_{p+s}$-Galois cover branched only at $\infty$ where it has upper jump $\sigma=h^{\prime} / m^{\prime \prime}$ written in lowest terms. Then $\sigma$ satisfies these necessary conditions: $\sigma>1 ; p \nmid h^{\prime}$; and $m^{\prime \prime} \mid(p-1)$.

Corollary 5.6. Suppose $2 \leq s<p$ and $\operatorname{gcd}(p-1, s+1)=1$. Then all but finitely many rational numbers $\sigma$ satisfying the necessary conditions of Notation 5.5 occur as the upper jump of an $A_{p+s}$-Galois cover of $\mathbb{P}_{k}^{1}$ branched only at $\infty$.

Proof. Theorem 4.9 implies that there exists an $A_{p+s}$-Galois cover of $\mathbb{P}_{k}^{1}$ branched only at $\infty$ with upper jump $\sigma_{s}=(p+s) /(p-1)$. The condition on $s$ implies that $\sigma$ is written in lowest terms and thus $m^{\prime \prime}=p-1$. The corollary then follows from Lemma 5.3 and Theorem 5.4 .

\subsection{Realizing lower jumps for $A_{n}$-Galois covers with inertia $\mathbb{Z} /(p)$}

Question 5.7. Suppose $G$ is a quasi- $p$ group whose order is strictly divisible by $p$. For which prime-to- $p$ integers $h$ does there exist a $G$-Galois cover 
$\pi: X \rightarrow \mathbb{P}_{k}^{1}$ branched only at $\infty$ with inertia group $\mathbb{Z} /(p)$ and lower jump $h$ ?

By Theorem [5.4, all sufficiently large prime-to- $p$ integers $h$ occur as the lower jump of a $G$-Galois cover of the affine line with inertia $\mathbb{Z} /(p)$. The question is thus how large $h$ needs to be to guarantee that it occurs as the lower jump of such a cover. In [5. Thm. 3.1.4], the authors prove that every prime-to- $p$ integer $h \geq p-2$ occurs as the lower jump of an $A_{p}$-Galois cover of the affine line with inertia group $\mathbb{Z} /(p)$. The next corollary improves on that result.

Corollary 5.8. Let $p \geq 5$. Let $h_{0}=(p+1) / \operatorname{gcd}(p+1,4)$. There exists an $A_{p}$-Galois cover of $\mathbb{P}_{k}^{1}$ branched only at $\infty$ with inertia group $\mathbb{Z} /(p)$ and lower jump $h$ for every prime-to-p integer $h \geq h_{0}$.

Proof. It suffices to prove that there exists an $A_{p}$-Galois cover of the affine line with inertia group $\mathbb{Z} /(p)$ and lower jump $h_{0}$; once this small value is realized for the lower jump of such a cover, then all larger prime-to- $p$ integers occur as the lower jump of such a cover by Theorem 5.4. Note that the upper and lower jumps are equal when the inertia group has order $p$.

Let $t=(p-1) / 2$. Then $\operatorname{gcd}(p-1, t(t-1))$ equals $(p-1) / 2$ if $p \equiv 1 \bmod 4$ and equals $p-1$ if $p \equiv 3 \bmod 4$. Consider the $A_{p}$-Galois cover $\pi_{t}: X_{t} \rightarrow \mathbb{P}_{k}^{1}$ in Theorem 4.6 which is branched only at $\infty$. If $p \equiv 3 \bmod 4$, then $\pi_{t}$ has inertia group of order $p$ and upper jump $(p+1) / 4$. When $p \equiv 1 \bmod 4$, then $\pi_{t}$ has inertia group of order $2 p$ and upper jump $(p+1) / 4$. In the latter case, taking $d=2$ in Lemma 5.3 yields an $A_{p}$-Galois cover of the affine line with inertia group of order $p$ and upper jump $(p+1) / 2$.

We now provide a partial answer to Question 5.7 for all other alternating groups whose order is strictly divisible by $p$.

Corollary 5.9. Let $2 \leq s<p$. If $p=7$, assume $s \neq 2$. Let $h_{s}=s(p+$ $s) / \operatorname{gcd}(p-1, s(s+1))$. There exists an $A_{p+s}$ Galois cover of $\mathbb{P}_{k}^{1}$ branched only at $\infty$ with inertia group $\mathbb{Z} /(p)$ and lower jump $h$ for every prime-to-p integer $h \geq h_{s}$.

Proof. By Theorem 4.9, there exists an $A_{p+s}$-Galois cover $\phi_{s}: Y_{s} \rightarrow \mathbb{P}_{k}^{1}$ branched only at $\infty$ with inertia group $G_{0}$ of order $p m_{s}$ and upper jump $\sigma_{s}=(p+s) /(p-1)$ where $m_{s}=(p-1) s / \operatorname{gcd}(p-1, s(s+1))$. Applying Lemma 4.1 with $r_{2}=m_{s}$ produces an $A_{p+s}$-Galois cover of the affine line with inertia group $\mathbb{Z} /(p)$ and lower jump $h_{s}$. This completes the proof by Theorem 5.4. 
Corollary 5.10. Let $p \neq 7$ be an odd prime. Let $h_{1}=2(p+2) / \operatorname{gcd}(p-1,3)$. There exists an $A_{p+1}$-Galois cover of $\mathbb{P}_{k}^{1}$ branched only at $\infty$ with inertia group $\mathbb{Z} /(p)$ and lower jump $h$ for every prime-to-p integer $h \geq h_{1}$.

Proof. By Theorem [4.9, letting $s=2$, there exists an $A_{p+2}$-Galois cover $\phi_{2}: Y_{2} \rightarrow \mathbb{P}_{k}^{1}$ branched only at $\infty$ with inertia group $G_{0}$ of order $p m_{2}$ and upper jump $\sigma_{2}=(p+2) /(p-1)$ where $m_{2}=(p-1) / \operatorname{gcd}(p-1,3)$. The lower jump $h$ of $\phi_{2}$ equals $(p+2) / \operatorname{gcd}(p-1,3)$.

Consider the $A_{p+1}$-Galois subcover $\tilde{\phi}: Y_{2} \rightarrow Z_{2}^{\prime}$ of $\phi_{2}$. It is branched above $P_{(\infty, 0)}$ where it has ramification index $m_{2}$ and above $P_{(\infty, \infty)}$ where it has ramification index $p m_{2} / 2$. The lower jump of $\tilde{\phi}$ above $P_{(\infty, \infty)}$ equals the lower jump $h$ of $\phi_{2}$. The upper jump of $\tilde{\phi}$ is thus $\tilde{\sigma}=2(p+2) /(p-1)$. Applying the Riemann-Hurwitz formula to $\phi_{2}$ and $\tilde{\phi}$, we note that $Z_{2}^{\prime}$ has genus 0 . Another way to see this is that the equation $g_{2}$ yields that $x=\left(y^{p+s}+1\right) / y_{s}$ and so the function field of $Z_{2}^{\prime}$ is $L_{2} \simeq k(y)$.

Thus $\tilde{\phi}$ is an $A_{p+1}$-Galois cover of the projective line branched at two points. Note that $\tilde{\phi}$ is disjoint from an $m_{2}$-cyclic cover of the projective line branched at $\{0, \infty\}$. Applying Lemma 4.1 with $r_{2}=m_{2}$ removes the tamely ramified branch point. In particular, it yields a Galois cover $\tilde{\phi}^{\prime}: Y_{2}^{\prime} \rightarrow \mathbb{P}_{k}^{1}$ branched only at $\infty$, with ramification index $p$. The upper (and lower) jump of $\tilde{\phi}^{\prime}$ is $\sigma^{\prime}=m_{2} \tilde{\sigma}$ which equals $2(p+2) / \operatorname{gcd}(p-1,3)$. This completes the proof by Theorem 5.4.

\subsection{Realizing small upper jumps for $A_{p}$-Galois covers}

The upper jump $\sigma=h / m$ of an $A_{p}$-Galois cover of the affine line satisfies the necessary conditions $\sigma>1, \operatorname{gcd}(h, m)=1, m \mid(p-1) / 2$, and $p \nmid h$. As a generalization of Question 5.7, we can ask which $\sigma$ satisfying the necessary conditions occur as the upper jump of an $A_{p}$-Galois cover of the affine line.

In [5. Thm. 2], the authors prove that all but finitely many $\sigma$ which satisfy the necessary conditions occur as the upper jump of an $A_{p}$-Galois cover of the affine line. That result generalizes both Corollary 5.8 (where $m=1$ ) and Corollary 5.1 (which can be rephrased as stating that all divisors of $(p-1) / 2$ occur as the denominator of $\sigma$ for such a cover). Specifically, given a divisor $m$ of $(p-1) / 2$ and a congruence value of $h$ modulo $m$, [5, Thm. 3.1.4] provides a lower bound on $h$ above which all $\sigma=h / m$ (satisfying the necessary conditions) are guaranteed to occur. The bound is $a(p-2)$ where $a$ is such that $1 \leq a \leq m$ and $a \equiv-h \bmod m$. 


\begin{tabular}{|c|c|c|c|}
\hline $\mathrm{p}$ & $\sigma$ obtained from [5, Thm. 3.1.4] & $\sigma$ unknown from [5] & Theorem $\underline{4.6}]$ \\
\hline 5 & $3,4,6, \ldots$ & 2 & None \\
& $3 / 2,7 / 2,9 / 2, \ldots$ & None & $2,3,4$ \\
\hline 7 & $5,6,8, \ldots$ & $2,3,4$ & \\
& $5 / 3,8 / 3,10 / 3, \ldots$ & $4 / 3$ & $3,4,5,6,7,8$ \\
\hline 11 & $9,10,12, \ldots$ & $2,3,4,5,6,7,8$ & $12 / 5$ \\
\hline \multirow{2}{*}{13} & $9 / 5,14 / 5,19 / 5, \ldots$ & $2,3, \ldots, 10$ & $3,4,5,6,7,8,9,10$ \\
& $11,12,14,15, \ldots$ & $3 / 2,5 / 2,7 / 2,9 / 2$ & $5 / 2,7 / 2,9 / 2$ \\
& $11 / 2,15 / 2,17 / 2 \ldots$ & $4 / 3,5 / 3,7 / 3,8 / 3,10 / 3$ & $10 / 3$ \\
& $11 / 3,14 / 3,17 / 3, \ldots$ & $7 / 6$ & \\
\hline
\end{tabular}

Theorem 4.6 improves on [5, Thm. 3.1.4] by providing some new values of $\sigma$ which were not previously known to occur as the upper jump of an $A_{p}$-Galois cover of the affine line. Corollary 5.8 is an example of that improvement; here are two more examples.

Example 5.11. Small primes: The first column of the table shows the values of $\sigma$ that are achieved in [5, Thm. 3.1.4]. The second column contains rational numbers satisfying the necessary conditions whose status was not known from [5]. The final column contains new values of $\sigma$ which are guaranteed to occur in Theorem 4.6.

Example 5.12. Suppose $p \equiv 1 \bmod 3$ and $m=(p-1) / 6$ and $h \equiv-1 \bmod$ $m$. Then the lower bound on $h$ to guarantee that $h / m$ occurs as the upper jump of an $A_{p}$-Galois cover of the affine line from [5, Thm. 3.1.4] is $p-2$ and from Theorem 4.6 is $(p-3) / 2$. Suppose $p \equiv 2 \bmod 3$ and $m=(p-1) / 2$ and $h \equiv-3 \bmod m$. Then the lower bound on $h$ to guarantee that $h / m$ occurs as the upper jump of an $A_{p}$-Galois cover of the affine line from [5, Thm. 3.1.4] is $3(p-2)$ and from Theorem 4.6 is $3(p-3) / 2$.

Proof. The previous lower bounds are a direct application of [5, Thm. 3.1.4]. For the new lower bounds, when $t=3$, then Theorem 4.6 states that $\sigma_{3}=$ $3(p-3) /(p-1)$ occurs as an upper jump of an $A_{p}$-Galois cover of the affine line. If $p \equiv 1 \bmod 3$, then $m=(p-1) / 6$ and $h=(p-3) / 2 ;$ (note that $h \equiv-1 \bmod m)$. If $p \equiv 2 \bmod 3$, then $m=(p-1) / 2$ and $h=3(p-3) / 2$; (note that $h \equiv-3 \bmod m$ ). 


\section{References}

[1] Shreeram Abhyankar. Coverings of algebraic curves. Amer. J. Math., 79:825-856, 1957.

[2] Shreeram S. Abhyankar. Galois theory on the line in nonzero characteristic. Bull. Amer. Math. Soc. (N.S.), 27(1):68-133, 1992.

[3] Shreeram S. Abhyankar. Resolution of singularities and modular Galois theory. Bull. Amer. Math. Soc. (N.S.), 38(2):131-169 (electronic), 2001.

[4] Irene I. Bouw. Covers of the affine line in positive characteristic with prescribed ramification. Accepted for publication to Fields Communications Volume WIN - Women In Numbers (Proceedings of the WIN Workshop, Banff International Research Station, Banff Canada), editors A. Cojocaru, K. Lauter, R. Pries, R. Scheidler.

[5] Irene I. Bouw and Rachel J. Pries. Rigidity, reduction, and ramification. Math. Ann., 326(4):803-824, 2003.

[6] David Harbater. Formal patching and adding branch points. Amer. J. Math., 115(3):487-508, 1993.

[7] David Harbater. Abhyankar's conjecture on Galois groups over curves. Invent. Math., 117(1):1-25, 1994.

[8] Jürgen Neukirch. Algebraic number theory, volume 322 of Grundlehren der Mathematischen Wissenschaften [Fundamental Principles of Mathematical Sciences]. Springer-Verlag, Berlin, 1999. Translated from the 1992 German original and with a note by Norbert Schappacher, With a foreword by G. Harder.

[9] Rachel J. Pries. Conductors of wildly ramified covers. II. C. R. Math. Acad. Sci. Paris, 335(5):485-487, 2002.

[10] M. Raynaud. Revêtements de la droite affine en caractéristique $p>0$ et conjecture d'Abhyankar. Invent. Math., 116(1-3):425-462, 1994.

[11] John Scherk. The ramification polygon for curves over a finite field. Canad. Math. Bull., 46(1):149-156, 2003. 
[12] Jean-Pierre Serre. Local fields, volume 67 of Graduate Texts in Mathematics. Springer-Verlag, New York, 1979. Translated from the French by Marvin Jay Greenberg.

[13] Henning Stichtenoth. Algebraic function fields and codes. Universitext. Springer-Verlag, Berlin, 1993. 